This item was submitted to Loughborough's Research Repository by the author.

Items in Figshare are protected by copyright, with all rights reserved, unless otherwise indicated.

\title{
Police response officer selection development of tool to aid the dispatch of police response officers
}

\section{PLEASE CITE THE PUBLISHED VERSION}

http://www.icores.org/

PUBLISHER

INSTICC / SCITEPRESS

VERSION

AM (Accepted Manuscript)

\section{PUBLISHER STATEMENT}

This work is made available according to the conditions of the Creative Commons Attribution-NonCommercialNoDerivatives 4.0 International (CC BY-NC-ND 4.0) licence. Full details of this licence are available at: https://creativecommons.org/licenses/by-nc-nd/4.0/

\section{LICENCE}

CC BY-NC-ND 4.0

\section{REPOSITORY RECORD}

Leigh, Johanna M., Lisa M. Jackson, and Sarah J. Dunnett. 2015. "Police Response Officer Selection Development of Tool to Aid the Dispatch of Police Response Officers". figshare.

https://hdl.handle.net/2134/17315. 


\title{
Police Response Officer Selection Development of Tool to Aid the Dispatch of Police Response Officers
}

\author{
Johanna Leigh $^{1}$, Sarah Dunnett ${ }^{1}$ and Lisa Jackson ${ }^{1}$ \\ ${ }^{1}$ Department of Aeronautical and Automotive Engineering, Loughborough University, Loughborough, United Kingdom \\ \{J.M.Leigh, S.J.Dunnett, L.M.Jackson\}@lboro.ac.uk
}

Keywords: $\quad$ Police Response, Vehicle Routing, Multi Objective, Decision Making, Simulation.

\begin{abstract}
It's essential the Police force use their resources to the highest possible efficiency to ensure adequate service in the face of major funding cuts. Automation of the response officer selection process can improve efficiency by assisting in selecting the most appropriate response officer to attend an incident. Currently dispatchers are tasked with selecting the appropriate response officers to send to incidents. This may not result in the most efficient officer being selected to attend an incident. Providing a software tool to assist in the decision making process will decrease uncertainty in the decision and hence increase the likelihood of the most efficient officer being selected to attend an incident. The selection considers response time, availability, area coverage, driving standard and traffic conditions. The tool is specific to the police dispatch process and hence accounts for factors which are not which general included in other dispatch tools.
\end{abstract}

\section{INTRODUCTION}

The UK police force is facing major funding cuts which have led to an increasing focus on improving the running efficiency of the service to ensure the reduction in funding does not result in a decrease in quality of service. One main area of concern is the incident response service, as reducing the quality of this service would lead to a reduction in public safety. To keep this service running efficiently, with reduced funding, improvements must be identified. One means of increasing efficiency is to ensure that the most appropriate officer is sent to an incident. Currently dispatchers must make a fast decision on which officer must attend each incident using the information available. Due to a lack of information the process does not always lead to the most efficient officer being chosen. The work outlined in this paper identifies the appropriate selection criteria for response officers and uses this information to develop a tool which can be used by dispatchers to select the most appropriate response officer.

The tool consists of three major elements, mapping, routing and decision making. The mapping tool shows the road map of the geographical location of interest. The routing tool uses the map to determine the routes officers can take to incidents, this enables the officer who would reach the incident in the shortest time to be identified. In determining these routes the tool currently takes into account the types of roads and predicted traffic conditions using road weightings. The decision tool then determines the most suitable officer to attend an incident taking into account many factors, as when selecting an officer the quickest route is not the sole consideration. Other considerations include officer availability, effects on area coverage and drivers driving qualification, these are all considered in the current decision tool. The tool aims to decrease uncertainty in the dispatcher's decision making process and increase the probability of selecting the most appropriate officer. It is developed specifically for police officer dispatch by considering details such as effects of driving qualification and form of transport.

In this paper initially a background to the present study is given and past relevant work is discussed. The three elements of the officer selection tool identified previously and how they are implemented in the tool developed here are then described in more detail. The final stage of the paper details how discrete event simulation is used to test the effects the tool has on the dispatch process. The results can be verified by comparing them to the current process for selecting an officer to attend an incident.

Details of policing activities have been taken from Leicestershire police. In other police forces processes may vary slightly. 


\section{BACKGROUND}

When incidents are reported they are assigned a grade which is typically between one and four. Grades one and two require an officer to be sent within a certain time limit. Grades three and four can be dealt with over the phone or by a scheduled appointment. Grade one incidents are emergencies which require response within fifteen minutes. Incidents that qualify include when there is a danger to life or immediate threat of violence. Grade two incidents are priority situations which require response within sixty minutes. Incidents which qualify include those where a caller is vulnerable.

Police officers are assigned to grade one and two incidents by a dispatcher. The dispatcher allocates the police officers as they see fit. When allocating officers to incidents they aim to minimise the response time and increase availability and area coverage. Response time is defined as the time between the call being answered and officers reaching the location of the incident. The availability is defined as the time officers are free to patrol. Area coverage is the number of officers able to reach a location within response time guidelines. Dispatchers often don't have all the information to make an informed decision of which officer to send to an incident and hence ask officers who can attend. This method typically does not result in the most suitable officer being selected for maximised efficiency of resources.

Current research in this area is limited. There are many other services and businesses which dispatch resources to locations. These include other emergency services, maintenance engineers and taxis. There is more extensive research in these areas of dispatch than in police dispatch. These studies are relevant but cannot be used due to the specific decisions involved in police officer selection.

\subsection{Emergency Service Dispatch}

Due to its importance there has been some previous research into the emergency service dispatch process. The majority of this work has considered the ambulance and fire services.

The ambulance service faces similar issues to the police force as they need to deploy their ambulances to incidents with the highest efficiency. Their aim is to reach each situation in the shortest period of time. Extensive studies have been carried out to ensure efficient positioning of ambulances, for example (Henderson \& Mason, 2004), (Haghani et al, 2003) and (Bandara et al, 2013). The study by (Henderson
\& Mason, 2004) states that the ambulance service also lacks a dispatch support tool. In this study decisions were made on dispatching ambulances and locating ambulances and stations. The ambulance service planning software 'BARTSIM' was developed to analyse information and simulate results. The software was implemented in an ambulance service in Australia.

(Haghani et al, 2003) developed a dispatch tool for the ambulance service. The tool included; flexibility in assignment to allow reallocation of resources as conditions changed, real time traffic information and weightings to cases depending on their priority, to give preference to attending more severe incidents. The computational time of this process was a major issue as results are required quickly in emergency situations.

The recent study on the ambulance service by (Bandara et al, 2013) explained the difficulties incurred in dispatching the appropriate ambulance to attend an incident. It explains that the closest ambulance should not always be selected as it is not always optimal to aim to minimise the average response time. Also ambulances shouldn't be assigned on a first-come-first-served basis. When considering selection the severity of the incident and the effects on area coverage should be considered. In this case the area coverage is measured by the number of demand points that can be met by the paramedic units within the set response time.

A relevant study into the fire service dispatch process by (Ignall et al, 1982) looked to reduce the second vehicle response to severe incidents requiring more than one fire engine. Historical data was used to predict how many fire engines would be required.

\section{METHOD}

The tool developed here has three sections; a mapping system, route finder and decision maker. The mapping system details the possible paths which can be travelled on. The route planner finds the appropriate routes between officers and incidents. The decision tool selects the officer depending on factors such as availability and driving standard. These sections will now be described in more detail.

\subsection{Mapping}

In order to enable effective officer selection it is necessary to have a road network model in which officers and incidents can be positioned and routed. 
The road information needs to be adaptable to allow information to be changed such as the speed possible along roads. To allow for this adaptability a road map will be created using information available from OpenStreetMaps (OpenStreetMaps, 2014) such as the longitudinal and latitude points along roads. From this information it is possible to model the map as a directed graph using Equation 1:

$$
\mathrm{G}=(\mathrm{V}, \mathrm{E})
$$

This equation uses a group of vertices (V) to represent the longitude and latitude points given as points along a road and uses edges (E) to represent the roads which join the vertices. Figure 1 shows how this is represented in graph form. The graph is directed because roads may be one-way and hence can only be travelled in one direction; this direction is represented by the arrows.

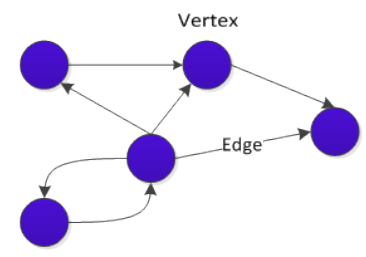

Figure 1: Directed graph.

Each edge has an associated weighting to account for the cost of travelling on the edge. The cost in this case is time, which will be considered when finding the appropriate route to travel.

From the data provided vertices are plotted and joined by the edges to create roads. This is used to develop a map for the entirety of the area of interest. Figure 2 is the road map of Leicestershire produced and Figure 3 is a zoned in section of Leicester centre plotted. The colour of the road illustrated the type of road, further information can be found in the key. Information such as direction of the roads and weightings are contained within matrices within the mapping tool.

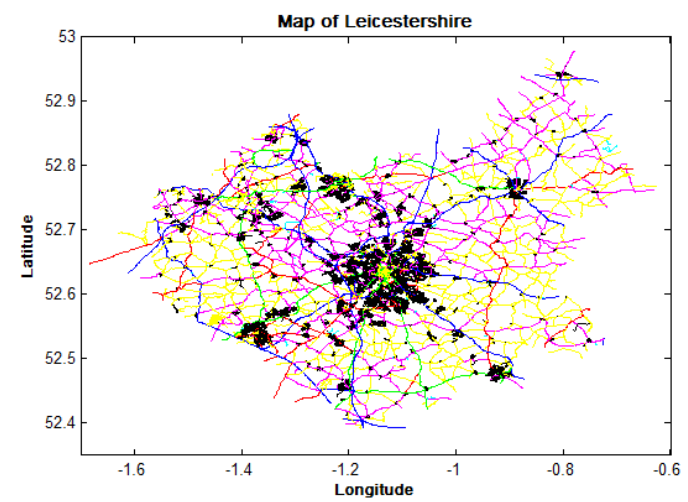

Figure 2: Road map of Leicestershire.

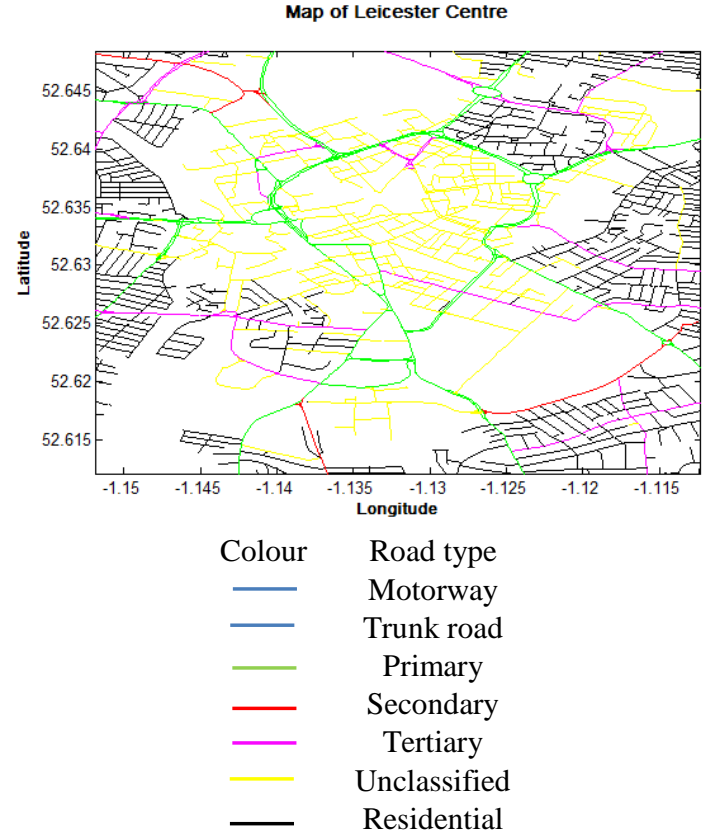

Figure 3: Map of central Leicester.

To navigate through the road system a method of finding a path from a current location to a target location is required, this is found through routing which is detailed in the next section.

\subsection{Routing}

This section of the tool is to determine which officer could reach an incident in the shortest time. In an emergency the shortest response time is the main objective for routing. Current methods of deciding which officer to send to an incident consider the closest officer which isn't necessarily the quickest.

The predicted time for each officer to reach the incident of concern is calculated by finding the quickest path from officer to incident using Dijkstra's algorithm. The possible paths considered are detailed on the map. The time taken to travel along each section of the path is calculated using the distance travelled along the road and the weighting given to the road. The road weighting considers type of road and predicted traffic and is discussed further in section 3.3.4.

The route from every officer in Leicestershire to an incident is not calculated. To save computational time the officer must meet certain criteria to be included in the routing process. For example, in an emergency situation officers which are unavailable, as they are busy with other duties, are excluded from the search. Of those available the n closest officers 
to the incident are found using simple 'as a crow flies distance' and these are the officers who are considered. $\mathrm{n}$ in this case is taken to be four but requires further testing to find the optimal value. Figure 4 shows the route from an officer to an incident determined by the tool. The route chosen utilises main roads due to the road weighting system.

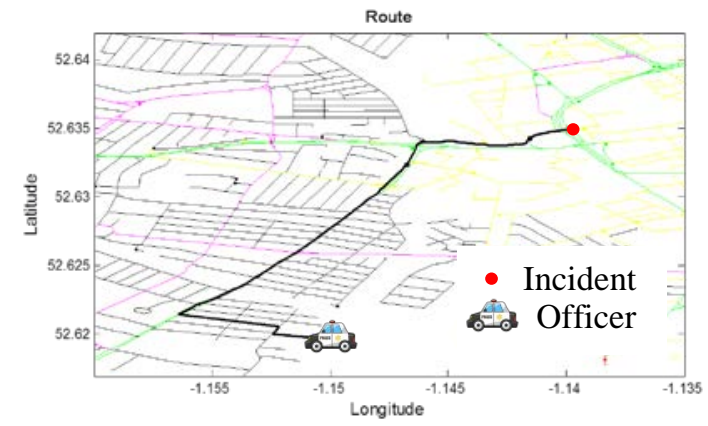

Figure 4: Route from officer to incident.

The quickest route isn't the only consideration when selecting an officer. Other criteria are considered in the decision making tool.

\subsection{Decision Criteria}

The decision of which officer to select to attend an incident is based on multiple factors including the officer location, the officer availability and driving standard, grade of the incident and area coverage at the officer location. How each of these factors impact the decision is discussed further in the following subsections. Figure 5 shows the two different selection criteria for emergency and priority response.

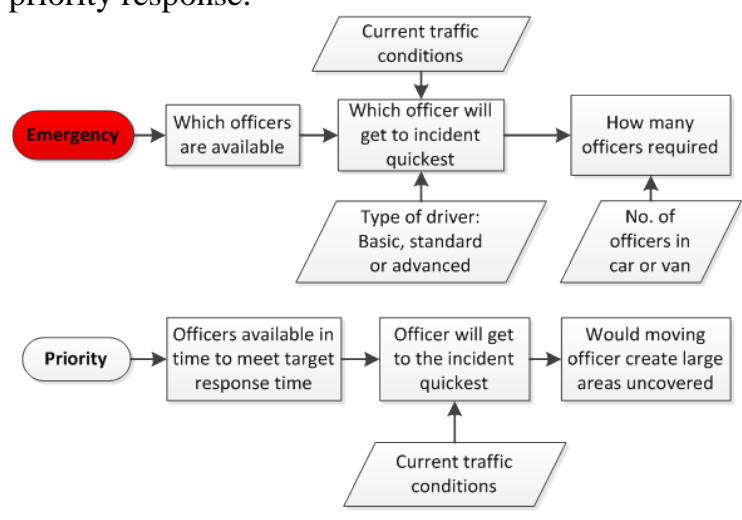

Figure 5: Selection criteria.

The emergency situation decision considers availability and the predicted response times accounting for traffic and driving standard. The priority situation considers availability, predicted response time accounting for traffic and area coverage.

\subsubsection{Response Times}

The grade of an incident determines the response time target. The response time targets are set by the government as fifteen minutes for a grade one and sixty minutes for a grade two, where grade one is an emergency situation and grade two is a priority situation. All other grades are sorted on the phone or by a scheduled meeting. The tool developed here is useful for incidents classed as grade one and grade two, as these both require dispatchers to send an officer in a timely response.

\subsubsection{Availability}

Officers have varying availability status markers depending on what they are currently undertaking. These can include available, attending to incident, break, etc. Available officers are ready to be sent to perform a task. Officers attending to incident are not available and the time they become available is hard to predict, officers on a break are unavailable though the time they become available can be predicted.

The status markers are used to determine whether an officer can be selected to attend an incident. In emergency situations a response is required immediately hence available officers are the only option. In priority situations response is not required immediately hence officers who are predicted to become available in the time window can also be selected. Of all the status markers, officers on a break and those processing prisoners are the two for which the time at which they become available can be predicted. The use of this factor as a selection criterion depends on the reliability of officers remembering to update their status.

\subsubsection{Driving Standard}

There are currently three main driving standards for police officers. There are basic drivers; which allows the officer to drive at the road speed limit. There are standard drivers; which allows the driver in emergency situations to use blue lights and sirens and go above the given speed limit (typically limited to 20mph over). And finally there are advanced drivers who can do the same as a standard driver with the addition of pursuing cars which fail to stop. Hence in an emergency situation a standard or advanced driver who is further away from the incident than a basic driver may be able to reach the 
incident quicker. Therefore in an emergency situation, when predicting the time taken to travel the route, the driving qualification of the officer driving is considered. The road weightings for a basic driver remain the same as in a priority situation. The road weightings for standard and advanced drivers are reduced to account for the increase in speed and parting of traffic. These road weightings are discussed further in section 3.3.4.

\subsubsection{Road Weightings}

Road weightings are applied to roads to give preference to travelling on particular roads and give a fair indication of how long each route will take to travel for comparison with other officer's routes. These weightings allow road type and traffic to be accounted for. This is necessary as road type determines the speed which vehicles can travel on the road under normal road conditions and the traffic conditions determine the speed which vehicles can travel down a road during congested times. The distance travelled along the road is multiplied by the road's weighting to give a more accurate prediction of the time taken to travel along the road. Road type has a constant effect on the road due to speed limits and hence the weighting associate with this is constant. Traffic varies depending on many factors such as time of day, day of the week, season and accidents and hence traffic weightings will vary depending on these factors. Accidents can't be predicted and hence won't be accounted for in the weighting system.

Different weightings are given officers on foot or bike so they can also be considered in the decision process. Whilst routing paths are considered where in vehicles they are not considered.

\subsubsection{Area Coverage}

Area coverage is the level of officers who are present in an accessible location to the area considered. The area coverage of a road is measured by the ability of an officer to reach it within the set response time limits. This officer doesn't have to be available, only present in the area where coverage is required. The level of coverage in an area can be determined by how many of the officers could reach a road within the limits of the response time. For example if two vehicles could reach the road the area coverage is two. The possible demand is determined using historical data in each area. Those areas with a demand of one should maintain an area coverage level of one.
When selecting an officer to attend an incident the dispatcher should seek to maintain appropriate area coverage and not remove all officers from an area. Within the tool it will recommend not moving officers if a large area will be left uncovered.

\subsection{Simulation}

The use of simulation allows the effects of using the tool developed to be analysed before implementation into a dispatch team. This is vital as it's too high risk to implement into the police force without validation.

To simulate the use of the officer selection tool a model of the process which requires the tool is set up. The simulation runs through a period of time where incidents occur and uses the tool to decide which officer should attend each of these incidents. Information input into the model such as incident data etc has been taken from historical data from Leicestershire Police. These scenarios test the decision making capabilities of the tool. The results of the decision are to be compared to real life statistics for average incident response times and officer availability.

The simulation is a discrete event simulation where an event is an incident occurring. The incidents are at variable time increments hence it is a variable time step simulation. This means the simulation will skip periods where incidents are not occurring which leads to a more efficient simulation.

Figure 6 shows the simulation process. Initially the map of the area of interest is created using data from OpenStreetMaps, following this the testing period is set and incident data for this period is generated. The incidents then trigger the simulation to run the decision making process to determine which officers to send to the incident. The selection runs through different criteria depending on whether the incident is classed an emegency or priority incident by the system. When making a decision, information from the resource list is used to determine where officers are currently located and their status and driving ability. Once an officer is selected their status is updated on the resource list and the next incident is inserted into the simulation. For the purpose of the simulation the time the officer will be undertaking this task is set using historical information of that type of incident. When using the tool in the field the officer status will be set to 'attending incident' until they update their status. The simulation records information on response times, distance travelled by each officer and availability for the purpose of analysis. 


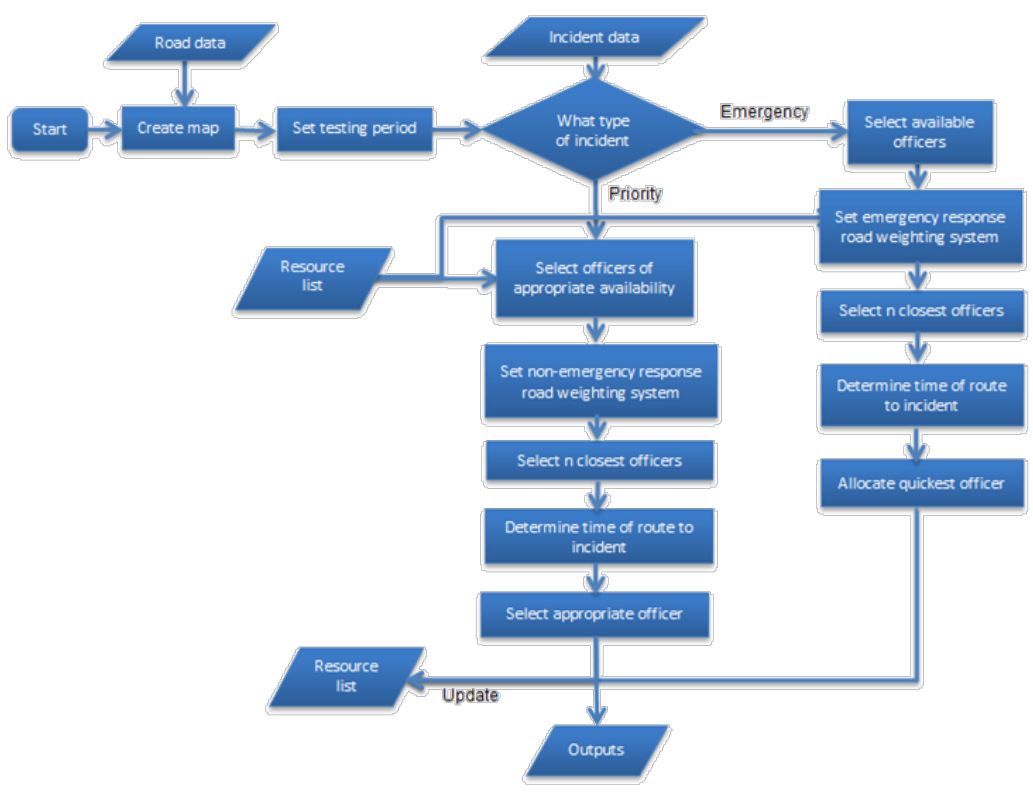

Firgure 6: Simulation process.

\section{RESULTS}

Preliminary findings from the simulation show significant differences in efficiency between using the decision tool to determine which response officer should attend an incident and selecting a random officer to attend an incident. The response times are lower, distance travelled by vehicles is lower and availability is increased. This shows an increase in efficiency in the police response service.

\section{CONCLUSION}

A need for an officer dispatch tool has been expressed by multiple police forces. To identify this issue a tool has been developed which can be used to select the most appropriate officer to send to an incident. The tool takes into account factors which are not currently used in the dispatch process which improve the likelihood of selecting the most efficient officer. The initially results show an increase in officer efficiency and prove this tool worthy of further development and implementation.

\section{FUTURE WORK}

Further work will include improvement to road weightings by using live traffic information to set weightings. This will give a more accurate idea of the traffic conditions and allow accidents to be accounted for. Also a queuing process to priorities series incidents will be included.

\section{ACKNOWLEDGEMENTS}

The cooperation of the Leicestershire police force is gratefully acknowledged as without this support this project would not be possible. This work was supported by the Economic and Social Research Council [ES/K002392/1].

\section{REFERENCES}

Bandara, D., Mayorga, M., McLay, L., 2012. Priority dispatching strategies for EMS systems. Journal of the Operational Research Society, Volume 65, Issue 10.

Hanghani, A. Hu, H., Tian, Q, 2003. An Optimization Model for Real-Time Emergency Vehicle Dispatching and Routing. Transport Research Board.

Henderson, S., Mason, A., 2004. Ambulance Service Planning: Simulation and Data Visualisation. International Series in Operations Research and Management Science, Volume 70.

Ignall, E., Carter, G., Rider, K., 1982. An Algorithm for the Initial Dispatch of Fire Companies. Management Science, Volume 28, Issue 4.

OpenStreetMaps \& Contributors. (2014). Maps. Available: http://www.openstreetmap.org/. Last accessed 01/09/2014. 